\title{
Two dimensional simulation on immunoassay for a biosensor with applying electrothermal effect
}

\author{
Chih-Kai Yang, Jeng-Shian Chang, ${ }^{\text {a),b) }}$ Sheng D. Chao, ${ }^{\text {a),c) }}$ and Kuang-Chong Wu \\ Institute of Applied Mechanics, National Taiwan University, Taipei 106, Taiwan
}

(Received 12 June 2007; accepted 23 August 2007; published online 12 September 2007)

\begin{abstract}
For diffusion-limited proteins, the diffusion boundary layer on the reacting surface hinders the binding reaction. The authors performed finite-element simulations of the electrothermal effect on the reaction kinetics of $C$-reactive protein (CRP)-anti-CRP. The induced vortices stir the flow and enhance the transport rate of analytes. They attribute the enhancement to the reduction of the thickness of diffusion boundary layer. Significant interference patterns of the votices are observed by varying the position of the reacting surface. These patterns are utilized to optimize the enhancement factor, yielding 5.166 and 3.744 times for association and dissociation, respectively, under voltage $\left(15 \mathrm{~V}_{\mathrm{rms}}\right)$ and frequency $(100 \mathrm{kHz})$. ( 2007 American Institute of Physics.
\end{abstract}

[DOI: $10.1063 / 1.2784941]$

In recent years, the study of biosensors has become an active research field for health care use. It brings many interesting applications of microtechnology in the area of biochemical and biophysical systems. The development of immunoassay is to place emphasis on the high sensitivity and the real-time detecting ability for different mechanism-based biosensors, such as the microcantilever beam based biosensor, the surface plasmon resonance sensor, and the quartz crystal microbalance sensor. Although the basic principles of detection are different, these sensors involve the same kinetics of specific recognition of binding of the analyte and the ligand. The concentration of the formed analyte-ligand complex during the chemical binding is the major quantity correlating with the measured data from various sensors, and reflects the concentration of the analyte in the flow, which is the physical quantity most concerned in clinic applications. The specific recognition of analytes and immobilized ligands occurs at the reacting surface of a biosensor, which is a solidliquid interface. The reaction kinetics can be described as a two-step process ${ }^{1}$ namely, the mass-transport process and the chemical reaction process. When the analyte takes a longer time to transport by convection and diffusion to the reacting surface than chemical reaction, the whole reaction is restrained by mass-transport limitation and a diffusion boundary layer is formed on the reacting surface. ${ }^{2}$ Existence of such a diffusion boundary layer on the reacting surface would hinder the binding reaction from association and dissociation, yielding difficulties in practical applications of biosensors. Sigurdson et al. ${ }^{3}$ have found that the electrothermal microstirring effect in a microchannel is useful to enhance the binding efficiency for diffusion-limited molecules. They performed the calculation showing only the initial process of the association reaction without or with the electrothermal effect and gave the enhancement factor by comparing the initial slopes of the binding reaction curves.

In this paper, we perform a two dimensional full timespan simulation of the association and dissociation in a biosensor immunoassay with the sample of $C$-reactive protein

\footnotetext{
a) Authors to whom correspondence should be addressed.

${ }^{b)}$ Electronic mail: jschang@spring.iam.ntu.edu.tw

${ }^{c)}$ Electronic mail: sdchao@spring.iam.ntu.edu.tw
}

(CRP) pairs to predict the surface concentration of the analyte-ligand complex versus time relationship. The induced electrothermal force by the ac electric field can cause a vortex field which will reduce the thickness of the diffusion boundary layer and significantly increase the reaction rate to accelerate both the association and dissociation rate processes. Interference to the vortex field due to the existence of the reaction surface at various positions in the microchannel could cause different degrees of enhancement for association and dissociation, and will be discussed below.

In this work, we consider a two dimensional 500 $\times 150 \mu \mathrm{m}^{2}$ microchannel in the $x-y$ plane, containing a reacting surface of $40 \times 3 \mu \mathrm{m}^{2}$ in the middle of the bottom side and a pair of electrodes on the top opposite to the reacting surface [see Fig. 1(a)]. The buffer solution mixed with the analytes flows from left to right. The inlet is set at $x=0$. On the reacting surface are the immobilized ligands.

Temperature gradients occur as a result of Joule heating due to the nonuniform ac electric field. The gradient of temperature $T$ in the liquid causes inhomogeneities of the permittivity $\varepsilon$ and the conductivity $\sigma$ of the medium, which, in turn, gives rise to the forces causing fluid motion. The body force $\mathbf{F}_{E}$ is given by Ref. 4.

$$
\mathbf{F}_{\mathbf{E}}=-\frac{1}{2}\left[\left(\frac{\nabla \sigma}{\sigma}-\frac{\nabla \varepsilon}{\varepsilon}\right) \cdot \mathbf{E} \frac{\varepsilon \mathbf{E}}{1+(\omega \tau)^{2}}+\frac{1}{2}|\mathbf{E}|^{2} \nabla \varepsilon\right],
$$

where $\tau=\varepsilon / \sigma$ is the charge relaxation time and $\omega$ is the angular frequency of the electric field $\mathbf{E}$. The local variations in temperature change the gradients of conductivity and permittivity,

$$
\begin{aligned}
& \nabla \varepsilon=(\partial \varepsilon / \partial T) \nabla T, \\
& \nabla \sigma=(\partial \sigma / \partial T) \nabla T .
\end{aligned}
$$

We consider the quasistatic electric field which is the root mean square (rms) value of the ac field. The electrostatic field $\mathbf{E}$ is related to the electrical potential $\Phi$ by $\mathbf{E}=-\nabla \Phi$, where $\Phi$ satisfies the Laplace equation. A small amount of Joule heating could give rise to a temperature increase in the fluid. In order to estimate the temperature rise for a given electrode system, the following energy balance equation must be solved, ${ }^{5}$ 


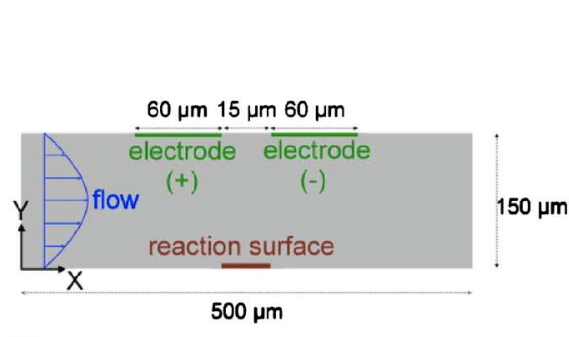

(a)

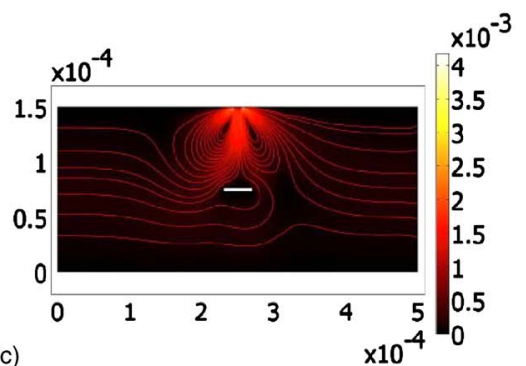

$$
\rho c_{p} \frac{\partial T}{\partial t}+\rho c_{p} \mathbf{V} \cdot \nabla T=k \nabla^{2} T+\sigma|\mathbf{E}|^{2}
$$

where $\rho, c_{p}, \mathbf{V}$, and $k$ are the density of the fluid, specific heat, velocity of the fluid, and thermal conductivity of the fluid, respectively. Here, $\sigma|\mathbf{E}|^{2}$ is the Joule heating as a source term. The fluid is assumed to be incompressible. The equation of motion is

$$
\rho \frac{\partial \mathbf{V}}{\partial t}+\rho \mathbf{V} \cdot \nabla \mathbf{V}-\eta \nabla^{2} \mathbf{V}+\nabla p=\mathbf{F}_{\mathbf{E}},
$$

where $\eta$ is the dynamic viscosity of the fluid and $p$ is the pressure. It is also assumed in this work that $\rho$ and $\eta$ of the modeled incompressible fluid are constant independent of temperature and concentration. Transport of analytes to and from the reacting surface is assumed to be described by the Fick's second law with convective terms,

$$
\frac{\partial[A]}{\partial t}+u \frac{\partial[A]}{\partial x}+\nu \frac{\partial[A]}{\partial y}=D\left(\frac{\partial^{2}[A]}{\partial x^{2}}+\frac{\partial^{2}[A]}{\partial y^{2}}\right),
$$

where $[A]$ is the concentration of analyte and $D$ is the diffusion coefficient of analyte, respectively. The reaction between the immobilized ligand and the analyte is assumed to follow the first order Langmuir adsorption model. ${ }^{2,6}$ During the reaction, the analyte-ligand complex $[A B]$ increases as a function of time according to the reaction rate equation,

$$
\frac{\partial[A B]}{\partial t}=k_{a}[A]_{\text {surface }}\left\{\left[B_{0}\right]-[A B]\right\}-k_{d}[A B],
$$

where $[A]_{\text {surface }}$ is the surface concentration of the analyte at the reacting surface by mass transport, $\left[B_{0}\right]$ is the surface concentration of the ligand, $[A B]$ is the surface concentration of the analyte-ligand complex, $k_{a}$ is the association rate constant, and $k_{d}$ is the dissociation rate constant.

In an immunoassay experiment, phosphate buffer saline is usually used to be a neutral buffer solution $(p \mathrm{H}=7.2)$, which is mixed with analytes as a carrier fluid. The fluid in the microchannel can be assumed that its physical properties are similar to water. The relative permittivity $\varepsilon_{r}$ and the electrical conductivity $\sigma$ are 80.2 and $5.75 \times 10^{-2} \mathrm{~S} \mathrm{~m}^{-1}$, respectively. The applied voltage is $15 \mathrm{~V}_{\mathrm{rms}}$ peak to peak with an Downloaded 21 Dec 2008 to 140.112.113.225. Redistribution subject
FIG. 1. (Color online) (a) Sketch of the model. The $x-y$ labeling is applied to Figs. 1 and 2. The flow field when applying a voltage of $15 \mathrm{~V}$ for different positions of the reacting surface are (b) $(250,1.5)$, (c) $(250,75)$, and (d) $(281,133)$, respectively. operating frequency of $100 \mathrm{kHz}$. Boundary conditions are $\Phi= \pm \mathrm{V}_{\mathrm{rms}} / 2$ at the two electrodes and electrically insulated elsewhere. The fluid in this domain is assumed to have properties as water. The density, specific heat, and thermal conductivity are $10^{3} \mathrm{~kg} / \mathrm{m}^{3}, 4184 \mathrm{~J} /(\mathrm{kg} \mathrm{K})$, and $0.6 \mathrm{~W} /(\mathrm{m} \mathrm{K})$, respectively. Boundary conditions are $T=300 \mathrm{~K}$ at both of the two electrodes and thermally insulated elsewhere. The value of the dynamic viscosity $\eta$ is set as that of water $10^{-3} \mathrm{~Pa}$. Since the flow in the microchannel is in the low Reynolds number condition, it is assumed as a laminar flow. The average velocity of the parabolic profile is set to $u$ $=10^{-4} \mathrm{~m} / \mathrm{s}$ at the inlet. Boundary conditions are $p=0$ at the outlet and nonslip elsewhere. The diffusion coefficients of human CRP $\left(=2.175 \times 10^{-11} \mathrm{~m}^{2} / \mathrm{s}\right)$ is obtained from Ref. 7 The inlet concentration of analyte is chosen as $[A]=6.4 \mathrm{nM}$. The initial surface concentration $\left[B_{0}\right]$ is assumed as 1.4 $\times 10^{-8} \mathrm{~mole} / \mathrm{m}^{2}{ }^{8}$. The diffusive flux at the reacting surface should be balanced against the reaction rate,

$$
-D\left(\frac{\partial[A]}{\partial y}\right)_{\text {surface }}=k_{a}[A]_{\text {surface }}\left\{\left[B_{0}\right]-[A B]\right\}-k_{d}[A B],
$$

where $k_{a}$ and $k_{d}$ for CRP-anti-CRP protein pairs can be found in Ref. 9 namely, $1.0 \times 10^{7} \mathrm{M}^{-1} \mathrm{~s}^{-1}$ and $2.6 \times 10^{-2} \mathrm{~s}^{-1}$, respectively. The initial conditions for both the concentrations of the analyte in the bulk $[A]$ and the concentrations of the analyte-ligand complex on the reacting surface $[A B]$ are all zero, respectively.

All the simulations have been performed using the finite element analysis software, COMSOL MULTIPHYSICS ${ }^{\mathrm{TM}}$ version 3.3 (COMSOL Ltd., Stockholm, Sweden). We simulate the dissociation phase of the binding reaction by terminating the supply of the analyte at a time after the binding reaction is saturated. The time of discontinuing the analyte is set manually. The results have been examined to be mesh independent by means of a normal convergence test.

The electrothermally driven stirring flow field would construct a pair of vortices, which can enhance the binding reaction of the protein pair by a strong convection of the analyte. Figure 1 shows the flow velocity fields in the microchannel with the ac voltage applied, when the reacting surface is located at three different locations, namely, $(250,1.5)$, to AIP license or copyright; see http://apl.aip.org/apl/copyright.jsp 

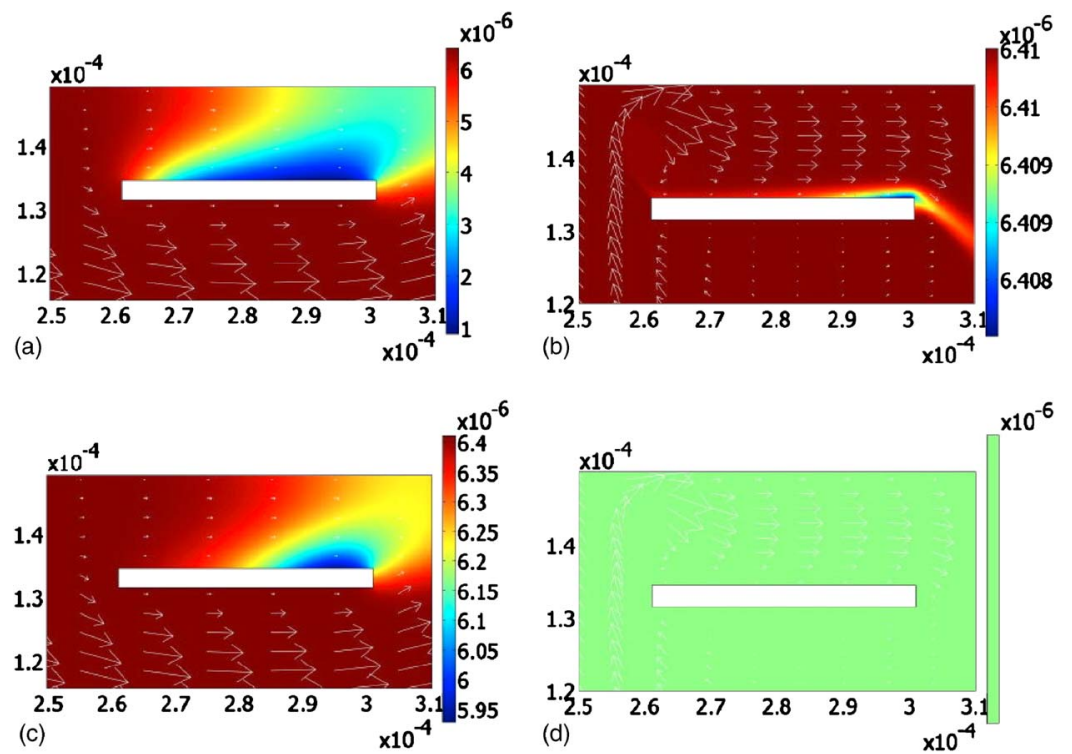

FIG. 2. (Color online) Development of the diffusion boundary layer of the CRP binding reaction without (left panel) or with (right panel) applying voltage where the reacting surface is at $(281,133)$. [(a) and (b)] Time $=500 \mathrm{~s}$. $[(\mathrm{c})$ and (d) $]$ Time $=1500 \mathrm{~s}$. Notice that the density scales are different to increase the plot visibility
$(250,75)$, and $(281,133)$. Notice that the length unit is micrometer. It is observed that the vortices are deformed to different degrees when the reacting surface is located at different locations. In particular, when the reacting surface is located near the negative electrode, $(281,133)$, a "squeezing" effect causes the fluid to flow rapidly through the channel between the top boundary and the reacting surface, which raises largely the speed of association and dissociation on the reacting surface. Location $(281,133)$ is the optimal position selected from about 30 sample locations to yield the best enhancement in association and dissociation in the present calculation.

For a diffusion-limited protein (the analyte), the consumption of $[A]_{\text {surface }}$ in the association phase is faster than the supply from the bulk. Thus, a diffusion boundary layer of the analyte is formed on the reacting surface, representing the lack of the analyte within the layer. The diffusion boundary layer also occurs in the dissociation phase when the supply of analyte in the flow is discontinued. And now the diffusion boundary layer represents a thicker concentration of the analyte than that in the bulk. It is found that the electrothermally driven stirring vortices have an advantage to decrease the thickness of the boundary layer, especially at the

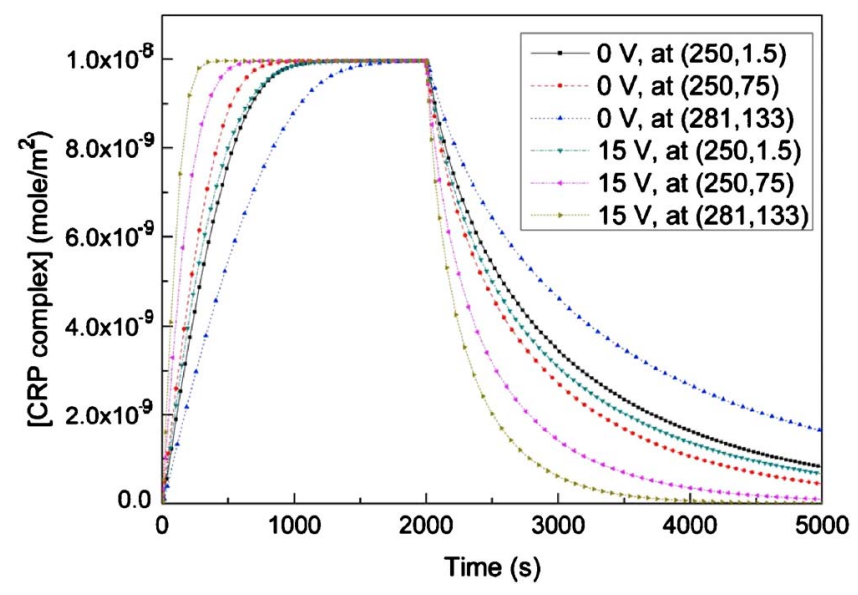

FIG. 3. (Color online) Surface concentration of CRP complex as a function of time with or without applying voltage for the locations showing in Fig. 1. position $(281,133)$, which is near to the negative electrode. Figure 2 presents the concentration field of diffusion boundary layer at the location $(281,133)$ without or with electrothermal effect considered at 500 and $1500 \mathrm{~s}$, respectively. It is observed that at $500 \mathrm{~s}$, the thickness of the diffusion boundary layer with applied ac electric field is largely reduced, compared to that without the applied field; and at $1500 \mathrm{~s}$, the boundary layer with the field applied is completely removed and the saturation for the association, in fact, reaches this moment. It is this reduction of the thickness of the boundary layer which enhances the reaction rates in terms of both the association and dissociation processes.

The simulated binding reaction curves for CRP-antiCRP with or without applying the electrothermally driven vortices are shown in Fig. 3 for three different locations of reacting surface. The initial slope is a good indicator describing the speed of reaction. We define the enhancement factor as the ratio of the initial slope of the binding reaction curve with applying electric field to that without applying the electric field. It is found that the enhancement is higher in the association than dissociation. The best enhancement factor is about 5.166 times for the association and 3.744 times for the dissociation, with respect to the field-free condition, at the position $(281,133)$ for the current configuration of the microchannel under study.

This research was supported by the National Science Council in Taiwan (NSC 96-2120-M-002-014). We acknowledge the National Center for High-Performance Computing (NCHC) for providing the computational resources.

${ }^{1}$ N. Camillone, Langmuir 20, 1199 (2004).

${ }^{2}$ D. B. Hibbert and J. J. Gooding, Langmuir 18, 1770 (2002).

${ }^{3}$ M. Sigurdson, D. Wang, and C. D. Meinhart, Lab Chip 5, 1366 (2005).

${ }^{4}$ A. Ramos, H. Morgan, and A. Castellanos, J. Phys. D 31, 2338 (1998).

${ }^{5}$ L. D. Landau and E. M. Lifshitz, Fluid Mechanics (Pergamon, London, 1959), p. 188.

${ }^{6}$ I. Langmuir, J. Am. Chem. Soc. 40, 1361 (1918).

${ }^{7}$ Y. Hokama, M. K. Coleman, and R. F. Riley, J. Immunol. 95, 156 (1965).

${ }^{8}$ E. Behravesh, V. I. Sikavitsas, and A. G. Mikos, Biomaterials 24, 4365 (2003).

${ }^{9}$ C. Chou, H. Y. Hsu, H. T. Wu, K. Y. Tseng, A. Chiou, C. J. Yu, Z. Y. Lee, and T. S. Chan, J. Biomed. Opt. 12, 024025 (2007). 